the expression of nPTB in the developmental transition from myoblast to muscle cell ${ }^{10}$.

Understanding how PTB and nPTB mediate splicing regulation should make it possible to define the arrangement of RNA sequence motifs that each of these factors recognizes (that is, their 'splicing codes') and to predict which additional exons are responsive to these factors. We are not there yet. Certain arrangements of C/U-rich motifs in the flanking introns have been shown to signify PTB-regulated exons, but less is known about how such motif codes differ for nPTB. The good news is that the wealth of microarray data now in hand provides the opportunity to decipher some of the fine points of the splicing codes for PTB and $\mathrm{nPTB}$. The bad news is that researchers unraveling these codes are likely to meet with complications. PTB or $\mathrm{nPTB}$ may regulate other splicing factors that induce changes in exon skipping or inclusion in response to RNA motifs independent of the
PTB and nPTB splicing codes. In addition, the involvement of coregulators could potentially magnify or even reverse the functions of PTB or nPTB. nPTB has been shown to interact with and functionally antagonize the neuronal splicing factor Nova in splicing reporter assays, indicating that this possibility should be considered ${ }^{5}$. Interestingly, some of the exons in the microarray analysis of Boutz et al. ${ }^{2}$ showed atypical changes, indicating a positive role for PTB or nPTB.

In a crucial window during mammalian embryonic development, precursor neuroblasts stop dividing and migrate to different parts of the brain to become diverse types of neurons. There are nearly 10,000 types of neurons, and diversity has been seen even within neuronal subtypes ${ }^{11}$. The question is irresistible: what are the roles of PTB and $\mathrm{nPTB}$ in specifying the functions and fates of these diverse neuronal populations in the brain? And what happens when things go wrong in Alzheimer's disease, schizophrenia, epilepsy and brain malignancies? There is enough here to capture scientists imagination for a long time to come.

\section{COMPETING INTERESTS STATEMENT}

The author declares no competing financial interests.

1. Lipscombe, D. Curr. Opin. Neurobiol. 15, 358-363 (2005).

2. Boutz, P.L. et al. Genes Dev. 21, 1636-1652 (2007).

3. Garcia-Blanco, M.A., Jamison, S.F. \& Sharp, P.A. Genes Dev. 3 12A, 1874-1886 (1989).

4. Patton, J.G., Mayer, S.A., Tempst, P. \& Nadal-Ginard, B Genes Dev. 5, 1237-1251 (1991).

5. Polydorides, A.D., Okano, H.J., Yang, Y.Y., Stefani, G. \& Darnell, R.B. Proc. Natl. Acad. Sci. USA 97, 6350-6355 (2000).

6. Markovtsov, V. et al. Mol. Cell. Biol. 20, 7463-7479 (2000).

7. Wollerton, M.C., Gooding, C., Wagner, E.J., Garcia-Blanco, M.A. \& Smith, C.W. Mol. Cell 13, 91-100 (2004).

8. Zhu, B., Ramachandran, B. \& Gulick, T. J. Biol. Chem 280, 28749-28760 (2005)

9. Lim, L.P. et al. Nature 433, 769-773 (2005).

10. Boutz, P.L., Chawla, G., Stoilov, P. \& Black, D.L. Genes Dev. 21, 71-84 (2007).

11. Muotri, A.R. \& Gage, F.H. Nature 441, 1087-1093 (2006).

\title{
An ESCRT for daughters
}

During normal cell division, cytokinesis begins as sister chromatids separate during mitotic anaphase. Constriction of the cell membrane by actin and myosin results in formation of the cleavage furrow, which further develops into a protein-rich membranous midbody structure. Abscission, the final stage of cell division, requires cleavage of the midbody and gives rise to two daughter cells. Cytokinesis requires substantial rearrangement of the plasma membrane, and abscission has been shown to require the vesicle-trafficking exocyst complex as well as components of the SNARE fusion machinery.

Viral budding, a process used by enveloped viruses such as HIV-1, includes a membrane cleavage event that is structurally similar to what occurs in daughter-cell separation. Viral late domain proteins use the Endosomal Sortin Complex Required for Transport-I (ESCRT-I) protein Tsg101 and the ESCRT-associated protein Alix to facilitate release of viral particles from the plasma membrane of the host cell. Alix and the ESCRT-I complex recruit ESCRT-III to promote membrane fission.

New work from Carlton and Martin-Serrano (Science, published online 7 June 2007, doi:10.1126/science.1143422) further investigates the physiological roles of these ESCRT proteins. The authors started by monitoring the cellular localization of Tsg101 during the cell cycle and found that Tsg101 moves to the midbody in the late stages of cell division, which suggests that it and related ESCRT proteins may be involved in cytokinesis. A survey of the human interactome revealed an interaction between Tsg101 and the centrosomal protein Cep55. Cep55 localizes to the midbody in the later cytokinesis stages and is required for abscission. The authors found that the proteins directly interact, and that Cep55 is required for Tsg101 localization to the midbody. In addition, they were able to map the Cep55-binding site to a proline-rich region of Tsg101. Deletion of this segment abolished Cep55 binding and Tsg101 recruitment to the midbody. Further screening experiments revealed that Cep55 also interacts with Alix and is required for Alix colocalization with Cep55 at the midbody.

The authors investigated what part, if any, Tsg101 and Alix play in cytokinesis. Depletion of Tsg101 or Alix proteins by RNA interference resulted in multinucleated cells (see left panel), indicative of a cytokinesis defect. A similar phenotype had been observed previously when Cep55 expression levels were knocked down (right panel). In addition, Carlton and Martin-Serrano found that overexpression of a dominant-negative mutant of VPS4, an ATPase that mediates ESCRT complex recycling, resulted in multinucleated cells, indicating that abscission had been inhibited. This same mutant also inhibits retroviral late domain activity. Overexpression of Tsg101, Alix or Cep55 resulted in disruption of viral budding and cytokinesis.

Overall, the data indicate that the cellular mechanisms involved in abscission, viral budding and multivesicular bud formation (for which the ESCRT complexes were first characterized) share common functional components. Furthermore, they suggest that the ESCRT machinery required for viral budding also mediates the membrane fission events needed in the final stages of cell division.

Michelle Montoya 